\title{
Dynamic hyperinflation and intrinsic PEEP in ARDS patients: who, when, and how needs more focus?
}

\author{
Heyan Wang ${ }^{1}$ and Hangyong $\mathrm{He}^{2^{*}}$
}

Dear editor,

We read with great interest of the report by Coppola and colleagues [1] about the presence and possible factors of dynamic hyperinflation and intrinsic positive endexpiratory pressure (PEEP) in severe acute respiratory distress syndrome (ARDS) patients. They suggested that in sedated, paralyzed ARDS patients without a known obstructive disease, the amount of intrinsic PEEP during lung-protective ventilation is negligible and does not influence respiratory mechanical properties. However, some details about who, when, and how for monitoring and managing dynamic hyperinflation and intrinsic PEEP in ARDS patients is still needed to be defined.

First, who needs more attention for monitoring dynamic hyperinflation and intrinsic PEEP with ARDS? During lung-protective ventilation in ARDS, when a respiratory rate (RR) up to a maximal of 35 breaths/min was needed to provide a minute ventilation that minimized hypercapnia and respiratory acidosis, the shortening of the expiratory time consequent to the higher $R R$ may generate substantial intrinsic PEEP [2, 3]. In the study by Coppola et al. [1], average RR was only 16 breaths/min. Therefore, severe ARDS patients with a high RR requirement for hypercapnia and respiratory acidosis are at high risk of dynamic hyperinflation and intrinsic PEEP, which needs more investigation.

The second question is when should we focus on dynamic hyperinflation and intrinsic PEEP with ARDS? In the article reported by Coppola et al. [1], only

\footnotetext{
*Correspondence: yonghang2004@sina.com;

Comment on: Silvia Coppola, Alessio Caccioppola, Sara Froio, Erica Ferrari, Miriam Gotti, Paolo Formenti and Davide Chiumello. Dynamic hyperinflation and intrinsic positive end-expiratory pressure in ARDS patients. Critical Care 2019, 23:375(https://doi.org/10.1186/s13054-019-2611-6. Published on: 27 November 2019)

This comment refers to the article available at https://doi.org/10.1186/ s13054-019-2611-6

${ }^{2}$ Department of Respiratory and Critical Care Medicine, Beijing Institute of Respiratory Medicine, Beijing Chao-Yang Hospital, Capital Medical University, No. 8 Gongren Tiyuchang Nanlu, Chaoyang District, Beijing 100020, China Full list of author information is available at the end of the article
}

paralyzed patients in the very early stage of ARDS were investigated. However, in deeply sedated ARDS patients without paralysis, the respiratory entrainment with reverse triggering may cause breath stacking in deeply sedated non-paralyzed ARDS patient, which led to volumes and pressures that were incompatible with lung-protective ventilation, and may lead to intrinsic PEEP and dynamic hyperinflation [4].

Finally, how to recognize and calculate intrinsic PEEP in patients with ARDS, especially in patients without neuromuscular blocking agents. In Coppola's report [1], intrinsic PEEP was defined as the total PEEP minus the external PEEP, and intrinsic PEEP decreased when external PEEP was raised. Expiratory flow limitation and airway closure may be two factors mainly responsible for the development of intrinsic PEEP in ARDS patients, and the response to a raising external PEEP might be due to airway closure and flow limitation at low PEEP and an airway opening pressure at high PEEP. Thus, reliability of the calculation for intrinsic PEEP in Coppola's study may not be valid under condition of flow limitation, which can occur in patients with ARDS. A measurement of intrinsic PEEP at zero PEEP should be more accurate. Furthermore, for patients with spontaneous breath, an increasing number of reports indicate that measurement of intrinsic PEEP can be obtained both with advanced monitoring systems (esophageal and gastric manometry, diaphragm electromyography, electrical impedance tomography) and, with some limitations, with simple airways occlusion maneuvers in patients with spontaneous breath [5].

Therefore, details about who, when, and how to investigate intrinsic PEEP and dynamic hyperinflation in ARDS patients are still needed to be evaluated. Further researches are needed for early recognition and better measurement of intrinsic PEEP and dynamic hyperinflation in this population. 


\title{
Authors reply to: "Dynamic hyperinflation and intrinsic PEEP in ARDS patients: who, when, and how needs more focus"

\author{
Coppola Silvia', Caccioppola Alessio², Froio Sara', Ferrari Erica², Gotti Miriam¹, Formenti Paolo' and \\ Chiumello Davide ${ }^{1,2,3^{*}}$
}

\author{
'Department of Anesthesia and Intensive Care, ASST Santi Paolo e Carlo, San Paolo University Hospital, Via Di \\ Rudinì, Milan, Italy \\ ${ }^{2}$ Department of Health Sciences, University of Milan, Milan, Italy \\ ${ }^{3}$ Coordinated Research Center on Respiratory Failure, University of Milan, Milan, Italy
}

We appreciate the time the authors have taken to read our recent article published in critical care on the dynamic hyperinflation and intrinsic positive end-expiratory pressure in ARDS patients [1].

The development of dynamic hyperinflation and intrinsic PEEP is usually generated by the presence of one or by a combination of these variables such as the expiratory flow limitation, the increase in expiratory resistance, the alteration in respiratory mechanics (resistance and compliance) and the ventilator setting (respiratory rate) $[3,6]$.

Concerning the respiratory rate in promoting the dynamic hyperinflation, we found that the respiratory rate in our population was very similar to that found by Bellani et al. in the "Lung Safe" study (18 vs. $18 \mathrm{bpm}$, respectively), thus we can assume that our data reflect the common management of ARDS patients [7].

We completely agree that the higher is the respiratory rate, the higher is the possibility to increase dynamic hyperinflation and the intrinsic PEEP [3, 6]. Our patients were sedated and paralyzed, thus there was no double trigger or any form of asynchrony which could have increased the tidal volume or minute ventilation; moreover, the ventilatory setting was maintained fixed throughout the entire study period. We measured the intrinsic PEEP by the occlusion of the airway at the end expiration for a period of 3-5 s, this pressure should represent the average pressure (static intrinsic PEEP) in the different lung regions. Although we did not measure the expiratory flow limitation based on the pressure volume loop, the intrinsic PEEP did not clinically change by increasing PEEP suggesting a minimal role of expiratory flow limitation in the development of intrinsic PEEP [8].

\section{Acknowledgements}

None.

\section{Authors' contributions}

$\mathrm{HW}$ and $\mathrm{HH}$ are responsible for the study design and writing, and this manuscript is approved by HH. Both authors read and approved the final manuscript.

\section{Funding}

None.
Availability of data and materials

Not applicable.

Ethics approval and consent to participate

Not applicable.

Consent for publication

Not applicable.

Competing interests

The authors declare that they have no competing interests.

\section{Author details}

'Department of Critical Care Medicine, The Sixth Hospital of Guiyang, Guiyang City, Guizhou Province, China. ${ }^{2}$ Department of Respiratory and Critical Care Medicine, Beijing Institute of Respiratory Medicine, Beijing Chao-Yang Hospital, Capital Medical University, No. 8 Gongren Tiyuchang Nanlu, Chaoyang District, Beijing 100020, China.

Received: 1 December 2019 Accepted: 17 December 2019 Published online: 23 December 2019

\section{References}

1. Coppola S, Caccioppola A, Froio S, Ferrari E, Gotti M, Formenti P, Chiumello D. Dynamic hyperinflation and intrinsic positive end-expiratory pressure in ARDS patients. Crit Care. 2019;23(1):375.

2. de Durante G, del Turco M, Rustichini L, Cosimini P, Giunta F, Hudson LD, Slutsky AS, Ranieri VM. ARDSNet lower tidal volume ventilatory strategy may generate intrinsic positive end-expiratory pressure in patients with acute respiratory distress syndrome. Am J Respir Crit Care Med. 2002;165(9):1271-4.

3. Richard JC, Brochard L, Breton L, Aboab J, Vandelet P, Tamion F, Maggiore SM, Mercat A, Bonmarchand G. Influence of respiratory rate on gas trapping during low volume ventilation of patients with acute lung injury. Intensive Care Med. 2002;28(8):1078-83.

4. Bourenne J, Guervilly C, Mechati M, Hraiech S, Fraisse M, Bisbal M, Roch A, Forel JM, Papazian L, Gainnier M. Variability of reverse triggering in deeply sedated ARDS patients. Intensive Care Med. 2019;45(5):725-6.

5. Grasselli G, Brioni M, Zanella A. Monitoring respiratory mechanics during assisted ventilation. Curr Opin Crit Care. 2019;25:000-000. Epub ahead of print. https://doi.org/10.1097/MCC.0000000000000681.

6. Marini JJ. Dynamic hyperinflation and auto-positive end-expiratory pressure. Am J Respir Crit Care Med. 2011 Oct 1;184(7):756-62

7. Bellani G, Laffey JG, Pham T, Fan E, Brochard L, Esteban A, et al. Epidemiology, patterns of care, and mortality for patients with acute respiratory distress syndrome in intensive care units in 50 countries. JAMA. 2016;315(8):788-800.

8. Koutsoukou A, Armaganidis A, Stavrakaki-Kallergi C, Vassilakopoulos T, Lymberis A, Roussos C, et al. Expiratory flow limitation and intrinsic positive end-expiratory pressure at zero positive end-expiratory pressure in patients with adult respiratory distress syndrome. Am J Respir Crit Care Med. 2000 May;161(5):1590-6.

\section{Publisher's Note}

Springer Nature remains neutral with regard to jurisdictional claims in published maps and institutional affiliations. 